\title{
Neonatal Resuscitation: Practical Considerations
}

\author{
E. O. Ogboli and S. A. Eguma
}

Department of Anaesthesia, Ahmadu Bello University Teaching Hospital, Zaria, Nigeria.

Reprint requests to: Dr. E. O. Ogboli, Department of Anaesthesia, A. B. U. Teaching Hospital, Zaria, Nigeria.

\section{Introduction}

Neonatal resuscitation refers to the assistance of the newborn infant during the critical transition period from dependent foetal to independent neonatal life. ${ }^{1}$ Asphyxia at birth is predictable only $60 \%$ of the time. Approximately $6 \%$ of full-term newborns and up to $80 \%$ of those weighing $<1,800 \mathrm{~g}$ must be resuscitated in the delivery room. ${ }^{2}$ Every individual involved in providing care for mother and baby at delivery should have adequate knowledge and skills needed for neonatal resuscitation.

Resuscitation of the neonate starts during labour. ${ }^{3}$ Any compromise of the uteroplacental circulation readily produces foetal asphyxia. Intrauterine asphyxia during labour is the most common cause of neonatal depression. ${ }^{3}$ Foetal monitoring throughout labour is helpful in identifying which babies may be at risk, detecting foetal distress and evaluating the effect of acute interventions.

\section{Asphyxia and its Causes in the Newborn}

\section{Asphyxia}

The asphyxiated infant passes through a series of events:
- Rapid breathing and decrease in heart rate.

- Primary apnoea (most will resume breathing when stimulated).

- Irregular gasping decreased heart rate and decreased blood pressure.

- Secondary apnoea - once at this stage, they are unresponsive to stimulation.

Apnoea at birth should be treated as secondary apnoea and resuscitation commenced at once.

\section{Causes of Asphyxia in the Newborn}

In utero, there is no respiratory effort. At birth, breathing is initiated by no specific stimuli. Failure to breathe may be due to intrauterine asphyxia, analgesics or anaesthetic agents given to the mother during labour or delivery, prolonged labour, difficult delivery or immaturity (lack of surfactant). Other causes include:

- Maternal hypoxia.

- Trauma of labour.

- Placental infarction or premature separation, prolapse or knotti $\mathrm{g}$ of cord.

- Fetal respiratory failure, whic 1 may be;

1. Central, due to;

- Immaturity of the respiratory center. 
- Damage to the respiratory centre from trauma, diabetes or

- Oxygen lack from intrapartum fetal asphyxia.

- Narcotics and sedatives given to mother.

2. Peripheral, due to;

- Immaturity of the lungs.

- Respiratory obstruction.

- Muscular weakness.

- Fetal lungs full of liquor amnii or meconium.

- Intranatal pneumonia.

\section{Resuscitation of the Newborn}

Anticipation, adequate preparation and prompt initiation of neonatal resuscitation are critical for a successful outcome. An objective assessment of the newborn's cardiorespiratory status immediately at birth and during the first few minutes of life should influence decisions about the need for resuscitation. The Apgar scoring system is still the most widely accepted method for the identification of those newborns in need of cardiopulmonary resuscitation at birth. 4,5 Respiratory effort, heart rate, colour, muscle tone and reflex irritability are assessed. However, the need for resuscitation can be more rapidly assessed by evaluating the heart rate, respiratory activity and colour. ${ }^{1}$ There is no need to delay resuscitation until the complete Apgar score is available. The maximum score is 10 . The 1 -minute score correlates with survival, while the 5 -minutes score is related to neurologic outcome." A single clinical assessment at 1 minute does not distinguish between primary apnoea (which will usually recover without treatment) and terminal apnoea (when active resuscitation is required).

Grouping by the 1 minute Apgar score greatly facilitates resuscitation. (1)
Mildly asphyxiated neonates (Apgar score of 5-7) usually need only stimulation while $100 \%$ oxygen is blown across the face; (2) moderately asphyxiated neonates (Apgar score 3 -4) require temporary assisted positive pressure ventilation with mask and bag; and (3) severely depressed neonates (Apgar score of 0-2) should be immediately intubated, and chest compressions may be required.

The fundamentals of neonatal resuscitation are to establish and maintain a clear, secure airway, provide effective ventilation and support the circulation. Additional considerations include the thermal environment and the provision of energy, especially for the low birth weight infant. Resuscitation of the depressed neonate requires two or more persons - one to manage the airway and ventilation and another to perform chest compression, if necessary. A third person gently facilitates the placement of intravascular catheters and the administration of fluids or drugs. Because the most common cause of neonatal depression is intrauterine asphyxia, the emphasis in resuscitation is on respiration. ${ }^{3}$

\section{Initial Stabilisation}

\section{Prevent Heat Losses}

- Place infant under an overhead radiant heater.

- Dry body and head.

2. Open the Airway

- Position the infant supine or in lateral position with neck in neutral position and avoid over-extension, use mechanical suction with size $8 \mathrm{~F}$ or $10 \mathrm{~F}$ catheter. Pressure must not exceed $13.3 \mathrm{kPa}$.

- Limit suctioning to 5seconds at a time and monitor heart rate for bradycardia, which may follow deep pharyngeal stimulation. 
- If meconium is present, use a wider bore catheter.

3. Tactile Stimulation

- Slap or flick the soles of the feet.

- Rub the back gently.

If there is no response after 10 seconds, abandon tactile stimulation and assist ventilation." Evaluate the Infant's;

- Respiration.

- Heart rate if <100beats/minute, begin intermittent positive pressure ventilation (IPPV), heart rate $<60 \mathrm{~b} / \mathrm{m}$, begin chest compressions with administration of $100 \%$ oxygen.

- Colour.

\section{Airway}

The presence or absence of meconium in the amniotic fluid (10\% of deliveries) is an important determining factor. Thin watery meconium does not require suctioning. Thick (pea soup) meconium should be suctioned with a bulb syringe to avoid the apnoea and dysrrhythmias observed during catheter suction.

\section{Breathing}

Indications for oxygen and IPPV by facemask in the newborn include; 1) Central cyanosis; 2) bradycardia; 3) Failure to cry on stimulation. If facemask resuscitation is not successful then intubation will be required. Cyanosis will fail to respond to oxygen therapy in the presence of cyanotic heart disease, severe tracheooesophageal fistula, large diaphragmatic hernia and sever respiratory distress syndrome.

Indications for ventilation (IPPV) ${ }^{3}$ include:

- Apnoea.

- Heart rate less than 100 beats/minute.

- Persistent central cyanosis on $100 \%$. oxygen by facemask.
Assisted ventilation by bag and mask should be at a rate of $40-60$ breaths/minute with $100 \%$ oxygen. Adequacy of ventilation should be checked by auscultation and chest excursions.

Indications for intubation of the newborn:"

- Central cyanosis for more than 3 minutes.

- Bradycardia < 100 beats/minute for more than 3 minutes.

- Apnoea for more than 3 minutes.

- White asphyxia

(skin vasoconstriction).

- Cardiac arrest or depression.

- Apgar score of 0 - 2 .

To intubate a neonate, the patient should be on a flat table with no pillow. The head is put in a neutral position; the head should not be over-extended. A straight blade laryngoscope, such as a miller 0 or 1 or Magill's laryngoscope usually provides the best view. The laryngoscope handle is held with the thumb and index finger as the chin is supported with the remaining fingers. The straight blade of the infant laryngoscope should be inserted posterior to the epiglottis from the right side of the mouth and then lifted vertically, but gentleness is required. Pressure applied over the hypoid bone with the little finger will bring the larynx into view. A size $2.5,3.0$ or 3.5 tube should be used depending on the weight of the neonate. Intubation attempts should be limited to 20seconds to minimize hypoxia. Between attempts ventilate with bag and mask.

\section{Circulation}

The neonatal heart lies under the lower third of the sternum rather than the middle third. Two-thumb compression is the preferred method for external cardiac massage in the newborn infant. The neonate is held with both hands as 
each thumb is placed just beneath a line connecting nipples and the remaining fingers encircle the chest. The sternum is compressed $1 / 2$ to $3 / 4$ inches $(1 \mathrm{~cm})$ at a rate of $120 /$ minute. Chest compressions by the two-thumb method generate higher arterial and coronary perfusion pressures than the two-finger method. The aim should be to produce three chest compressions for every lung inflation.

\section{Medication}

For the majority of infants who require resuscitation, the only 'medication' needed will be $100 \%$ Oxygen delivered with effective ventilation. "The trachea is the most accessible route for the administration of drugs, and can be used for adrenaline, atropine, lidocaine and naloxone. ${ }^{2}$ However, absorption is incomplete, and the drug mass must be tripled in order to give amounts equivalent to the intravenous dosage. The umbilical vein can be used but the risk of haemorrhage and sepsis is high. The intramedullary route using a 20gurage spinal needle inserted into the proximal tibia or distal femur is feasible but the complications are numerous. The umbilical vein however remains the commonest route for giving drugs during major resuscitation.

- Adrenaline: remains the drug of choice in a cardiac arrest. 0.01 $0.03 \mathrm{mg} / \mathrm{kg} \quad(0.1-0.3 \mathrm{ml} / \mathrm{kg}$ of a $1: 10,000$ solution) should be given for asystole or a spontaneous heart rate of $<80$ beats/minute in spite of adequate ventilation and chest compressions. It can be repeated every 3-5 minutes.

- Naloxone: $0.01 \mathrm{mg} / \mathrm{kg}$ intravenously or $0.02 \mathrm{mg} / \mathrm{kg}$ intramuscularly, is given to reverse the respiratory depressant affect of opioids given to the mother in the last 4 hours of labour.
- Sodium bicarbonate: $(2 \mathrm{mg} / \mathrm{kg}$ of $0.05 \mathrm{mEq} / \mathrm{ml}$ solution) may be used in a prolonged arrest unresponsive to other medications or if profound metabolic acidosis is documented. It should be given slowly (over 2 minutes) to avoid intraventricular haemorrhage.

- Calcium: citrate intoxication only indicated in cases of hypokalaemia and hype-rmagnesaemia.

- Atropine: has a very minor role in neonatal resuscitation.

- Glucose: $200 \mathrm{mg} / \mathrm{kg}$ of a $10 \%$ solution is given only for documented hypoglycaemia because hyperglycaemia worsens hypoxic neurologic deficits. Also catecholamine release leads to hyperglycaemia. An infant who has low cardiac output despite adrenaline, volume expanders and

- Sodium bicarbonate may benefit from dopamine infusion, beginning with $50 \mathrm{~g} / \mathrm{kg} /$ minute and increasing to $200 \mathrm{~g} / \mathrm{kg} /$ minute as necessary.

\section{Fluids}

- Hypovolaemia must be suspected if the neonate remains pale, has a weak pulse or responds poorly to resuscitation.

- $5-10 \mathrm{ml} / \mathrm{kg}$ of placental blood + heparin $2 \mathrm{u} / \mathrm{ml}$.

- O-negative red cells, cross-matched mothers' blood.

- Albumin, 5\%.

- Lactated Ringer's solution.

- Normal Saline.

\section{Post Resuscitation Care}

Newborns who have been successfully resuscitated require close monitoring in a neonatal intensive care unit (NICU) or special care baby unit (SCBU). Post resuscitation care may include: 
- Arterial pH and blood gas estimation.

- Correction of documented metabolic acidosis.

- Use of volume expanders and/or vasopressors if hypotension persists.

- Appropriate fluid therapy.

- Screening for hypoglycaemia and hypocalcaemia.

- Chest x-rays for diagnostic purposes.

\section{Conclusion}

The key to successful resuscitation of the newborn infant is timely and appropriate intervention including oxygenation, ventilation, volume expansion and other therapies directed at relieving the distressed newborn. All health personnel involved in the delivery of babies must be familiar with the resuscitation of newborn infants. An organised educational programme in the standards and skills required for neonatal resuscitation is obligatory for any institution in which births occur and midwives and physicians involved must have access to such approved programmes.

\section{References}

1. James AG. Neonatal resuscitation, stabilization and emergency neonatal transportation. Intensive Care world 1994; 11:53 - 57.

2. Gung J. Foetal monitoring and neonatal resuscitation: What the anaesthetist should know. Can J Anaesth 1991; 38: R83 - R88.

3. Morgan GE jr., Mikhail MS. Obstetric anaesthesia In: Morgan GE jr., Mikhail MS (eds). Clinical anaesthesiology. Appleton \& Lange, Connecticut, 1996: 705 - 725.

4. Jain L, Vidyasagar D. Cardiopulmonary resuscitation of newborns. Pediatr Clin Nor Am 1993; 40: 287 . 302.

5. Apgar V. A proposal for a new method of evaluation of the newborn infant. Anesth Analg 1953; 32: 260 -268 .

6. Elliot RD. Neonatal resuscitation: The NRP guidelines. Continuing medical educatioin. Can J Anaesth 1994; 41: 742-753. 